Haike Meinhardt*

\title{
Leseforschung und ihr Potential für die bibliothekarische Leseförderung
}

https://doi.org/10.1515/bfp-2017-0044

Zusammenfassung: Die Entwicklung und Förderung von Lesekompetenz von Kindern wie Erwachsenen ist seit langem ein wichtiges Anliegen Öffentlicher Bibliotheken. Insbesondere im Rahmen der Bildungspartnerschaften haben Bibliotheken systematisch aufgebaute modulare Angebote für diesen Kompetenzbereich entwickelt. Die allermeisten Angebote der Öffentlichen Bibliotheken zielen dabei auf die Stärkung der Lesemotivation, um durch positive Leseerfahrungen die Leselust und damit durch eine erhöhte Lesehäufigkeit letztlich implizit die Lesekompetenz zu verbessern. Die Leseforschung hat jedoch in den letzten Jahren zu einem sehr differenzierten Verständnis des Leseprozesses und seiner Vorbedingungen geführt, dem die Entwicklung eines ebenso differenzierten didaktischen Instrumentariums folgte. Im Mittelpunkt dieses Ansatzes steht die Erkenntnis, dass der Komplexität des Leseprozesses nur ein entsprechend komplexes Leseförderungsmodell gerecht werden kann. Bibliotheken können von diesen Ansätzen vielfältig profitieren, wenn sie sich mit den Forschungsergebnissen vertraut machen und diese in die Entwicklung geeigneter Fördermaßnahmen umsetzen. Wenn Bibliotheken diese Herausforderung annehmen, könnte ihre Position als Lernort und Bildungspartner deutlich gestärkt werden.

Schlüsselwörter: Leseforschung; Öffentliche Bibliotheken; Lesekompetenz

\section{Reading Research and Its Potential for Reading Promotion in Public Libraries}

Abstract: One of the core undertakings of public libraries is, and since long has been, the fostering and development of reading skills of the young as well the as the adult. Building on partnerships in education, public libraries have systematically created modular offerings to promote this area of competencies. Most of these offerings target to develop a self-motivation for reading by creating a positive reading experience. It was believed, that an increased joy in reading, accompanied by a higher reading frequency

*Kontaktperson: Prof. Dr. Haike Meinhardt, haike.meinhardt@th-koeln.de would consequentially and implicitly lead to improved reading abilities. However, in recent years, as scientific research has reached a more sophisticated understanding of the reading process and its prerequisites, a more differentiated didactic tooling has been created. A central insight of this new approach is the actual complexity of the reading process in turn requires a more sophisticated methodology for the development of reading competencies. In utilising these new scientific findings to create suitable methods, public libraries can open up a multitude of opportunities for themselves. If libraries rise to the challenge, they can thoroughly strengthen their position as an institution of learning and education.

Keywords: Reading research; public libraries; reading literacy competencies

Inhalt

1 Veränderungsanstöße . . . . . . . . . . . . . . 319

2 Das lerndidaktische Lesekompetenzmodell von Rosebrock/Nix . . . . . . . . . . . . . . 321

2.1 Phonologische Informationsverarbeitung . . . . . 322

2.2 Phonologische Teilkompetenzen operationalisieren und in bibliothekarische Angebote transformieren . . . . . . . . . . . . . . . . . . 324

2.3 Graphem-Phonem-Korrespondenz und lokale

Kohärenzbildung................. 325

2.4 Förderung von Leseflüssigkeit. . . . . . . . . . 325

2.5 Herstellen von Globaler Kohärenz . . . . . . . . . 326

\section{Veränderungsanstöße}

Leseförderung bzw. (ausgeweitet auf die Vorläuferfähigkeiten) Sprach- und Leseförderung ist einerseits seit Jahrzehnten ein Kernbereich der Tätigkeit in Öffentlichen Bibliotheken sowie in Schulbibliotheken. Die Publikationen zum Thema sind vielfältig und zeigen in der Zusammenschau, ${ }^{1}$ dass sich sowohl der Kreis der Adressaten, den

1 Eine nur oberflächliche Recherche in den einschlägigen Datenbanken (DABI, LISTA ...) sowie im Katalog der DNB fördert zahlreiche 
die Bibliotheken erreichen wollen, immer weiter ausdifferenziert hat (Kindergartenkinder, Vorschulkinder, Schulkinder, weitere spezifische Zielgruppen wie Jungen, funktionale Analphabeten, Kinder bzw. Familien mit Migrationshintergrund etc.) als auch die bibliothekarischen Formate eine ungemeine Vielfalt aufweisen und längst die Integration digitaler Medien und Tools umgesetzt haben. Unter den Publikationen dominieren dabei eindeutig die praktisch ausgerichteten und auf Anwendung bzw. Transfer zielenden Titel und Beiträge, ${ }^{2}$ während die stärker theoretisch ausgerichteten Forschungsansätze noch Einzelfälle zu sein scheinen. ${ }^{3}$

Bekanntermaßen und wenig überraschend ist die Erforschung des Leseprozesses, die Entwicklung von Modellen der Lesekompetenz, die Analyse von Komponenten und Determinanten des Leseverständnisses und ein immens beackertes Forschungsfeld von (Literatur-)Pädagogen, Didaktikern sowie in der Entwicklungspsychologie, den Kognitions- und Sozialwissenschaften. Auch hier gibt es einen entsprechenden Output an Grundlagenliteratur, aber auch an lerndidaktischen und diagnostischen Materialien, ${ }^{4}$ vor allem im Hinblick auf eine stärker wissenschaftlich fundierte Praxis zur erfolgreichen Entwicklung von Lesekompetenz in Kindergärten und Schulen.

Und schließlich ist es die Bildungspolitik, die Impulse, Anlässe und Forderungen formulierte: zum einen im Kontext des Paradigmenwechsels hin zur Kompetenzorientierung und weg von einem Kanon zu vermittelnder Bildungsinhalte, denen in einer schnelllebigen und globalisierten Welt nur noch kurzfristige Geltung zugesprochen wird; 5 zum anderen durch die PISA-Untersuchungen, die eben genau diese Kompetenzen abprüfen, also problemorientierte Anwendungen (oder Scheinanwendungen) in den Mittelpunkt stellen und aus den Ergebnissen bildungspolitischen Handlungsbedarf formulieren. Für unseren Zusammenhang ist relevant, dass die für Deutschland vielfach als problematisch eingeschätzten Resultate im Bereich Lese-

Beiträge und Titel zutage, das soll als Beleg hier ausreichen. Alle ausgeführten Internetquellen wurden am 08.06.2017 überprüft.

2 Vgl. hierzu den sehr instruktiven Band von Brandt und Keller-Loibl (2015).

$3 \mathrm{Zu}$ nennen wäre hier die Wirkungsforschung in Hamburg: Vgl. dazu Krauß-Leichert und Paul (2014) oder auch die an Kompetenzkomponenten orientierte Untersuchung von Nathalie Hild im Kontext ihrer Bachelorarbeit (2016).

4 Vgl. dazu generell die Arbeiten und Studien von Andrea BertschiKaufmann, Norbert Groeben, Bettina Hurrelmann, Christine Garbe, Gudrun Schulz, Cornelia Rosebrock, Wolfgang Lenhard u. a.

5 Die vielfache Kritik an diesem Kompetenzparadigma sowie die mittlerweile sichtbaren negativen Auswirkungen können hier nicht Gegenstand sein; vgl. dazu Krautz (2016). kompetenz (als anerkannte Basiskompetenz) alarmiert haben, wobei vor allem die anhaltende Leseschwäche von etwa einem Viertel der Schülerschaft Besorgnis auslöste. Das wiederum führte verstärkt dazu, dass die Ergebnisse der Leseforschung aufgenommen und in Kompetenzstandards bzw. -stufen übersetzt wurden, die sich nun als schulisches „Gesetz“ nahezu flächendeckend in den Kerncurricula der einzelnen Bundesländer wiederfinden. Darüber hinaus kam es zu bildungspolitischen Initiativen wie „ProLesen. Auf dem Weg zur Leseschule“6 der KMK (Teilnahme 16 Bundesländer) oder auch Verbundforschungsprojekten wie „BISS: Bildung durch Sprache und Schrift““7

Auch wenn die Bibliotheken natürlich PISA zur Kenntnis genommen haben und die Notwendigkeit ihrer Anstrengungen auf dem Feld der Leseförderung vielfach daran rückkoppelnd artikulieren, ist das beschriebene Geschehen, die Übersetzung von Forschungsergebnissen in Kompetenzziele des Lesens, deren stringente Integration in die Curricula und die Umsetzung in pädagogische und didaktische Konzepte weitgehend an den Bibliotheken vorbei abgelaufen. ${ }^{8}$ Obwohl Bibliotheken in diesem Bereich seit Jahren intensive Bildungsarbeit leisten, stehen sie dennoch jedenfalls aus der Sicht der Bildungspolitik - an der Peripherie. Auch wenn sich Bibliotheken als Bildungspartner der Schulen sehen und manche sich in ihren Angeboten ausdrücklich an den formulierten Kompetenzzielen der Schulpläne der Länder ${ }^{9}$ orientieren, hat sich an diesem Befund wenig geändert. Ursächlich dafür ist aus meiner Sicht ganz entscheidend, dass in Sachen Lesekompetenzförderung nach wie vor ein Verständnis dominiert, das Lesen sozusagen als komplexe (vielleicht sogar amorphe) Gesamt-Fähigkeit ansieht, die vor allem gefördert werden soll, indem Freude, Spaß und Lust am Lesen geweckt werden, die zu wiederholten Leseerfahrungen ermutigen und so letztlich zu einer Habitualisierung des Lesens beim Kind beitragen soll. Unzweifelhaft ist dies ein richtiger und wichtiger Ansatz, aber er ist dennoch recht eindimensional und es ist an der Zeit, ihn zu erweitern und zu ergänzen.

Ein gewisser Druck, dies auch tatsächlich zu tun, resultiert aus einer weiteren relativ neuen, aber mit Nachdruck vorgetragenen Prämisse der Bildungspolitik. Es geht um die „Entdeckung“ des informalen und informellen Lernens, um die zunehmende Bedeutung von Lernprozessen, die außerhalb formaler Bildungskontexte, auch außerhalb von Kindergarten, Grundschule, weiterführender Schule

\footnotetext{
6 Vgl. ProLesen (2011).

7 Vgl. http://www.biss-sprachbildung.de/. Alle Internetquellen wurden am 8.6.2017 letztmalig geprüft.

8 Vgl. hierzu Hellenschmidt (2015) und Rose (2013).

9 Bspw. das MIZ Biberach, vgl. Raumel (2016).
} 
und Hochschule stattfinden. Umfassender Lernerfolg, so der gedankliche Ausgang, bedarf neben dem schulischen Lernen auch des stärker selbstgesteuerten und -bestimmten Lernens in Familie, Freundeskreis, in außerschulischen Bildungseinrichtungen etc. Es gilt, die Kompetenz der Bibliotheken „entlang der Bildungskette“10 fruchtbar zu machen. Dafür bedarf es auf Bibliotheksseite eines gezielteren und stärker ausdifferenzierteren Vorgehens. In diesem Zusammenhang sei darauf verwiesen, dass die Impulse dafür interessanterweise aus dem literaturpädagogischen Bereich kamen - dankenswert vor allem die Aktivitäten und Forschungsarbeit im Umkreis von Gudrun Marci-Boehncke (TU Dortmund, „Experten für das Lesen“ bzw. „Zertifikat „Literaturpädagogik“). ${ }^{11}$

Damit ist der Rahmen abgesteckt: Eine Leseforschung, die die Teilprozesse des Lesens analysiert hat, entsprechende Diagnoseinstrumente und Fördermethoden entwickelt hat. Eine Bildungspolitik, die diese Erkenntnisse in Kompetenzziele und -standards goss sowie in die Kindergärten- und schulischen Curricula implementierte und entsprechende Programme ins Leben rief - auch gerichtet an außerschulische Bildungspartner. Schließlich Erzieher und eine Lehrerschaft, die das alles in sinnvolle Alltagspädagogik umsetzen sollen. Und dann Bibliotheken, die in punkto Leseförderung bislang recht stark auf die bibliothekarisch erprobten und auf die neuen Medien adaptierten Ansätze setzen und davon ausgehen, dass „bibliothekarische Leseförderung [...] in erster Linie das Ziel [verfolgt], Lesefreude und Lesemotivationen auszubilden und in den verschiedenen Altersstufen zu stabilisieren" ${ }^{12}$

Im Folgenden soll aufgezeigt werden, wie diese differenten Bereiche stärker synthetisiert werden könnten ausgehend von einem sehr fruchtbaren Kompetenzmodell.

\section{Das lerndidaktische Lesekompetenzmodell von Rosebrock/Nix}

Wie oben bereits erwähnt, gibt es eine Fülle von Lesekompetenzkonzepten, ${ }^{13}$ die Lesekompetenz aus unterschiedlichen Blickwinkeln analysieren, mal stärker als kulturelle oder soziale Tätigkeit oder als kognitiven Prozess erfassen

10 Vgl. hierzu Eickelmann, Aufenanger und Herzig (2014).

11 Vgl. dazu zuletzt Marci-Boehncke und Trapp (2016) sowie Höft und Marci-Boehncke (2017).

12 Vgl. Brandt und Keller-Loibl (2015) 12.

13 Vgl. Garbe, Holle und Jesch (2010). und die sich nicht alle eignen, um den Bogen zur Praxis auch tatsächlich schlagen zu können.

Ein Modell, das dies in ausgezeichneter Weise leistet, ist das Kompetenzmodell, das Cornelia Roesebrock und Daniel Nix ${ }^{14}$ (ab 2008) entwickelt haben, um Lehrern eine praktikable Unterstützung für eine systematische schulische Leseförderung an die Hand zu geben und das viele der o.g. Perspektiven integriert. Lesekompetenz wird bei ihnen aus didaktischer Perspektive umfassend begriffen und dimensioniert als entwicklungsbedingter kognitiver Prozess, als subjektive Aktivität und als soziale Praxis. Jeder Dimension werden die entsprechenden Teilkomponenten und/oder Teilprozesse, die während des Lesens ablaufen, zugewiesen. Was Rosebrocks Modell so erfolgreich macht, ist die Zuordnung von diagnostischen Elementen sowie adäquaten Fördermethoden zu den entsprechenden Teilprozessen bzw. Komponenten. Der Lehrende erhält damit sozusagen das Gesamtpaket: die theoretischen Fundierung, die Diagnostik und die praktische „Lösung“ für den fördernden Unterricht. Da sich der Transfer für die bibliothekarische Praxis geradezu aufdrängt, soll das Modell im Einzelnen etwas genauer erläutert werden, wobei das Rosebrock'sche Modell hier etwas erweitert wird, da der bibliothekarische „Zugriff“" auf Lesekompetenz nicht erst mit dem Schulalter einsetzt, wie bei Rosebrock und Nix, sondern die sog. Vorläuferfertigkeiten seit geraumer Zeit integriert.

Der Ansatz für eine differenzierte Förderung ist mithin die Auffächerung all der Teilkomponenten, die für gelingendes Lesen notwendig sind, um die entsprechenden Lernprozesse gezielt zu adressieren: die Kenntnis darüber, in welchen Altersstufen welche Leseleistungen möglich sind, um bei der Förderung auch eine entwicklungsadäquate Passung zu erzielen und in welchen sozialen Kontexten Lesen im jeweiligen Entwicklungsverlauf vorrangig stattfindet.

Das Modell von Rosebrock und Nix zeigt (Abb. 2), dass Lesekompetenz keine Fähigkeit ,aus einem Guss“ ist, sondern einer Vielzahl von Teilfähigkeiten umfasst, die auf unterschiedlichen Ebenen angesiedelt sind: Diese betreffen die Kognition des Individuums - die kognitive Prozessebene; sie betreffen jedoch genauso Persönlichkeit und Identität des Lesenden - die psychosoziale Subjektebene und all das ist situativ eingebettet in soziale Situationen und Kontexte, beispielsweise schulische oder bibliothekarische. Hier spielt die soziale Ebene eine entscheidende Rolle (Abb. 2).

14 Vgl. Rosebrock und Nix (2014). Im Folgenden stütze ich mich ganz wesentlich auf die Ausführungen von Rosebrock und Nix. 


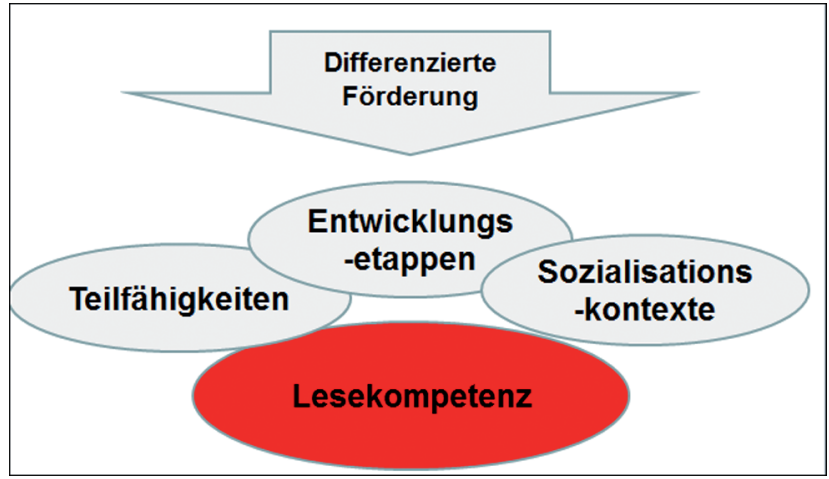

Abb. 1: Der Ansatz für eine differenzierte Förderung umfasst den Blick auf die Teilkomponenten des Leseprozesses entsprechend der jeweiligen kindlichen Entwicklungsetappen und die primären Sozialisationskontexte. Quelle: H.M. Vorlesungsskript

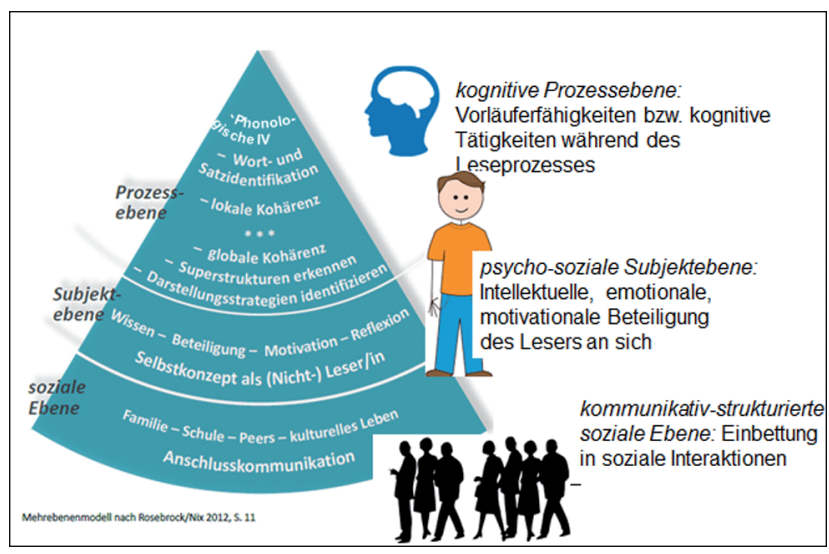

Abb. 2: Das Modell von Rosebrock und Nix, erweitert um die phonologische Informationsverarbeitung sowie mit ergänzenden Erläuterungen. Quelle: H.M. Vorlesungsskript

\subsection{Phonologische Informationsverarbeitung}

Das Zentrum des konzentrisch gedachten Kreisausschnittes (oder die Spitze des Tortenstückes) umfasst die Tätigkeiten während des Leseprozesses (s. Abb. 3), die als kognitive Verarbeitungsschritte notwendig sind, um überhaupt zu einem Textverstehen gelangen zu können. Schon dieser Bereich zeichnet sich durch eine Fülle von Verarbeitungsprozessen aus, von der phonologischen Informationsverarbeitung ${ }^{15}$ als wichtige sog. Vorläuferfähigkeit und zentralem Determinanten für den künftigen Prozess des Lesenlernens, bis hin zur Identifizierung von Darstellungsstrategien.

15 Im Folgenden nach Artelt et al. (2005).
Beginnen wir mit den Komponenten der phonologischen Informationsverarbeitung, die vom Lesenden geleistet werden müssen:

- Das ist zum einen die phonologische Bewusstheit (im weiteren Sinne), also die Fähigkeit zur Identifikation von größeren sprachlichen Einheiten wie Wörtern oder Silben, sie wird vor Schuleintritt erworben. In diesem Alter sollten Kinder die Fähigkeit erlangt haben, das einzelne Wort als solches, also als eine sprachliche Einheit zu erkennen und dieses Wort auch in Silben, als kleinere und zugehörige Einheiten, zerlegen zu können. Wenn die Entwicklung unproblematisch verläuft, erlernen Kinder diese Fähigkeit immanent mit dem wachsenden Hör- und Sprachvermögen.

- Zur phonologischen Informationsverarbeitung gehört auch die sprachliche Bewusstheit im engeren Sinne, auch phonemische Bewusstheit genannt. Dies ist die Fähigkeit zur Differenzierung (auch als Diskriminierung bezeichnet) von noch kleineren Einheiten - einzelnen Lauten; i.d.R. wird sie mit Beginn des schulischen Schriftspracherwerbs erworben, häufig aber auch schon vor Schuleintritt. Wenn ein Kind erkennt, dass das Wort „Baum“ mit einem B beginnt und einem $M$ endet, ist diese Verarbeitungsstufe erreicht. Sie ist wichtig, weil das Kind erst darüber überhaupt die Möglichkeit erhält, später die Laut-Buchstaben-Korrespondenzen $\mathrm{zu}$ erkennen, also das alphabetische Prinzip der Sprache zu verstehen.

- Das phonologische Rekodieren ist eine weitere Komponente. Hiermit ist die Fähigkeit gemeint, sich schriftliche Symbole (Grapheme) in ihre lautliche Entsprechung (Phoneme) zu übersetzen und auf das ,semantische Lexikon“ (das eigene Wort-BedeutungsWissen) zuzugreifen. Kinder, die gerade Lesen lernen, überführen beim lauten Lesen schrittweise eine Buchstabenkette in eine Lautkette, synthetisieren diese zum Wort und erkennen dann, ob dieses Wort Sinn macht (die Fähigkeit wird auch als „Leseinnenverständnis“"16 bezeichnet) oder ob es keinen Sinn macht (weil es ein Pseudowort ist oder den Kindern noch gänzlich unbekannt, also nicht Bestandteil des individuellen „Bedeutungslexikons“ ist). Zu Beginn, etwa mit dem ersten Schuljahr, braucht ein Kind häufig mehrere Leseversuche, bis es die Bedeutung eines Wortes ermittelt hat. Diese Fähigkeit ist für den Lesevorgang essentiell; hier muss mit der Zeit eine gewisse „Geschwindigkeit“ und „Automatisierung“ erreicht 
werden (weshalb in der Regel die Zeit gemessen wird, die der Rekodiervorgang benötigt), weil ansonsten

- das phonologische Rekodieren im Arbeitsgedächtnis gefährdet ist. Darunter ist zu verstehen, dass schriftliche Symbole, die Grapheme, im Arbeitsgedächtnis lautsprachlich repräsentiert bleiben müssen. Um bei o.g. Beispiel zu bleiben: Wenn das Kind die Grapheme eines Wortes mühsam in Laute übersetzt, muss es am Ende des Wortes noch wissen, für welchen Laut der Anfangsbuchstabe des Wortes stand, es muss also in der Lage sein, lautliche Repräsentationen sequentiell in seinem Kurzzeitgedächtnis halten zu können. Nur im Fall eines leistungsfähigen Arbeitsgedächtnisses bleiben bereits erlesene Wortkomponenten bis zur vollständigen Wort-Synthese aktiviert. ${ }^{17}$

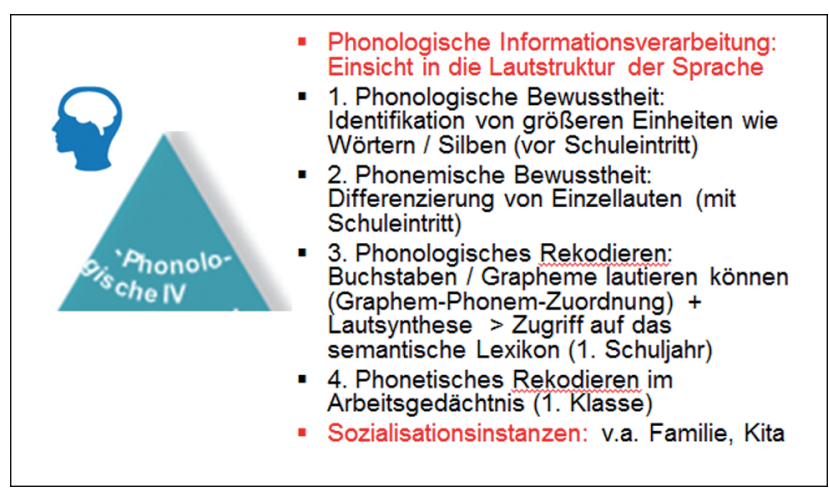

Abb. 3: Die Teilkomponenten der phonologischen Informationsverarbeitung sowie die Graphem-PhonemKorrespondenz. Quelle: H.M. Vorlesungsskript

Das phonologische Rekodieren verlangt bereits, dass Graphem-Phonem-Korrespondenzregeln gekannt und dem Vorgang zugrunde gelegt werden.

Bereits diese Aufgaben der phonologischen Informationsverarbeitung sind alles andere als einfach, binden beim Leseanfänger alle Ressourcen und verlangen höchste Konzentration und Anspannung. Kinder, die hier scheitern, haben kaum eine Chance, das Lesen je erfolgreich zu erlernen. Allerdings ist auch vielfach nachgewiesen, dass

17 Weitere Faktoren, die hier mit hineinspielen, sind natürlich der Wortschatz, die Fähigkeit, etwas korrekt und richtig auszusprechen (und zu hören) und sich zu artikulieren. Auch das Vorwissen über Schrift ist relevant: Kinder, die ein Begriff „vom Lesen“ allgemein haben (bspw. weil die Eltern oder ältere Geschwister lesen), entwickeln ein Gespür dafür, dass es eine Schriftsprache gibt, die mit der gesprochenen Sprache korrespondiert und zwar nicht dergestalt, dass die Schriftsprache gesprochene Dinge (Sachverhalte) eins zu eins abbildet, sondern in etwas anderes, eben Schriftzeichen, umsetzt. eine gezielte Förderung nachhaltig Verbesserungen bewirkt. ${ }^{18}$ Die Sozialisationsinstanzen, die dafür in erster Linie verantwortlich sind, sind - natürlich - die Familie, aber auch der Kindergarten.

Die gute Nachricht ist, dass die Diagnostik im Bereich der phonologischen Informationsverarbeitung weit entwickelt und wissenschaftlich abgesichert ist. ${ }^{19}$ Das sog. „Bielefelder Screening zur Früherkennung von LeseRechtschreibschwierigkeiten BISC“ oder auch das „Nürnberger Erhebungsverfahren zur phonologischen Bewusstheit“" (DFG-geförderte Forschungsprojekte) werden zur diagnostischen Früherkennung breit eingesetzt und machen durch die sehr differenzierten Erhebungsmethoden transparent, welche Teilkompetenzen der phonologischen Informationsverarbeitung beim betreffenden Kind unterentwickelt sind und wo sich eventuelle Risiken für das spätere Lesenlernen erkennen lassen, wenn bereits hier basale Fertigkeiten verfehlt werden.

Nun geht es natürlich nicht darum, dass Bibliothekare zu Diagnostikern werden, aber: sie sollten wissen, welche Teilkompetenzen in welchem Alter erwartet werden dürfen - hilfreich dafür sind bspw. die sog. diagnostischen Einschätzskalen. ${ }^{20}$ Sie könnten darauf aufbauend wesentlich gezieltere und sequentiell aufgebaute Förderungen entwickeln und diese den Kindergärten anbieten bzw. als informelle Lernorte Eltern und deren (Klein)Kinder adressieren - insbesondere natürlich Risikogruppen.

Um das zu verdeutlichen: Viele Bibliotheken offerieren sowohl für Klein- wie für Vorschulkinder Sprach- und Leseförderaktionen..$^{21}$ Die Stadtbibliothek Köln hat bspw. zwei Veranstaltungsformate geschaffen, die „Bücherbabys Sprach- und Sinnesförderung für die Kleinsten“ bis zwei Jahre und „Papalapap - für alle Pänz von drei bis sechs Jahren“. ${ }^{22}$ Beide Formate sind absolut sinnvoll und wie bei den meisten bibliothekarischen Angeboten geht es um eine niedrigschwellige erste und emotional positive gefärbte Begegnung mit Sprache und Medien in Vorlesesituationen, begleitet von musikalischen und motorischen Elementen, Fingerspielen, Reimspielen, mit viel Interaktion und $\mathrm{Zu}$ wendung.

18 Vgl. Barth (2012).

19 Vgl. dazu die Übersicht auf der Webseite des Deutschen Instituts für Internationale Pädagogische Forschung: http://www.biss-sprach bildung.de/biss.html?seite=122 sowie Barth (2012).

20 Vgl. Barth (2016).

21 Die Förderung der phonologischen Bewusstheit (auch in dieser Terminologie) hat m.W. als erste Bibliothek die Stadtbibliothek Brilon, Ute Hachmann, bereits vor vielen Jahren intensiv ins Programm genommen.

22 Vgl. http://www.stadt-koeln.de/leben-in-koeln/stadtbibliothek/ bildungsangebote. 
Auch die Angebote, die sich an die Erzieher selber richten, Aktionskisten und Aktionsvorschläge (mit Aktionsblättern als Handlungsvorschläge), gehen in diese Richtung ${ }^{23}$ und bieten ein buntes Potpourri von Anregungen: die „pure“ interaktiv gestalteten Vorleseaktion (Kniebücher), die Kombination einer Geschichte mit der Produktion von Geräuschen und Klängen („Conni macht Musik“), das malerische Umsetzen einer Geschichte („Käpten Knitterbart und seine Bande“), das freie Weiterreimen („Mit Ottern stottern, mit Drachen lachen“), Texträtsel („Die Olchis: so schön ist es im Kindergarten“), ein Buchstabenpuzzle und -quiz (,Das alleralbernste Abc-Buch“), daneben kleinere Spiele rund um das Erleben in der Bibliothek („Paula leiht sich was“).

Da ist allerhand dabei: die Motivationsförderung, das Training des Hörverstehens, die phonologische Bewusstheit auf verschiedenen Ebenen, der produktionsorientierte Umgang mit einem Text, das Textverstehen, die Förderung des Verständnisses von Schrift sowie die Phonem-Graphem-Zuordnung, dazwischen Einheiten, die eher die Vermittlung von Bibliothekskompetenz zum Ziel haben. Was nicht so leicht zu erkennen ist, ist das dahinter stehende Konzept: Gibt es einen systematischen Aufbau der Aktionen oder Kompetenzen? Erkennbar ist eher eine etwas beliebig erscheinende Zusammenstellung von sinnvollen Einzelbausteinen.

Um nicht missverstanden zu werden, das positive Erleben von Lese- und Mediensituationen, das Vorlesen selber sind wunderbare und ergiebige Fördermöglichkeiten, das ist vielfach nachgewiesen. Aber: Das reicht nicht immer und insbesondere dann nicht, wenn bereits Defizite sichtbar werden.

\subsection{Phonologische Teilkompetenzen operationalisieren und in bibliothekarische Angebote transformieren}

Wie könnte ein ergänzendes oder erweitertes oder stärker gezielteres Angebot aussehen. Ein Angebot, das zudem auch - terminologisch wie inhaltlich - erkennen lässt, dass die Bibliothek sich fachlich auf Augenhöhe mit ihren Kooperationspartnern bewegt und auf deren Anforderungen eingeht?
Eine probate Möglichkeit wäre es, die operationalisierten Anforderungen an die Teilkomponenten der phonologischen Informationsverarbeitung als solche bewusst $\mathrm{zu}$ machen und daraus Angebote zu entwickeln, die ein Lesetraining für diese basalen Fertigkeiten mit den im motivationalen Bereich liegenden Stärken von Bibliotheken verbinden.

Operationalisierte Teilkomponenten der phonologischen Informationsverarbeitung sind, phonologische Einheiten zu analysieren bzw. zu differenzieren und Veränderungen, Ersetzungen, Umstellungen, Auslassungen und Hinzufügungen von Phonemen durchführen zu können, bspw. ${ }^{24}$ Reime zu erkennen, Wörter segmentieren zu können, Wörter nach ihren Lauten vergleichen zu können, End- und Anlaute zu identifizieren, die Phoneme eines Wortes benennen zu können etc. Auch die Synthese von Phonemen gehört in diesen Anforderungsbereich: Silben zusammenzusetzen, Phoneme zu einem Wort zusammenzuziehen oder fehlende Phoneme in einem Wort ergänzen zu können etc.

Wenn es um das phonologische Rekodieren im Arbeitsgedächtnis geht, helfen Übungen, die generell darauf abzielen, verbal zu memorieren: das Nachsprechen von Wortklangbildern, die Wiedergabe lautlicher Sequenzen (auch Pseudoworte und Zahlenfolgen, bei Beibehaltung der serialen Ordnung), um die Gedächtnisspanne zu erweitern, also die auditive Merkfähigkeit und die auditive Aufmerksamkeit zu verbessern. Auch Übungen zur visuellen Wahrnehmung sind erwiesenermaßen hilfreich.

Die Stabilisierung und beständige Erweiterung des Wortschatzes, um beim phonologischen Rekodieren auf das semantische Lexikon zugreifen zu können, hat vor allem in diesem Bereich einen eminent positiven Einfluss. Hier ist es insbesondere das Vorlesen (und die diversen Variationen - wie bspw. Kamishibai), das dies leistet. Bibliotheken adressieren diese Teilkompetenz bereits in idealer Weise und vermitteln dabei dem Kind zusätzlich auch (meist) ein mentales Modell von Schriftsprache.

Für eine gezielte Förderung der Teilkomponenten der phonologischen Informationsverarbeitung gibt es eine Fülle von erprobten und kreativen Materialien, die sich häufig an Erzieher und Pädagogen richten. Als Grundlage für die Entwicklung eigener, auf die bibliothekarische Situation hin besser zugeschnittener Programme, sind sie jedoch sehr gut geeignet.
23 www.stadt-koeln.de/leben-in-koeln/stadtbibliothek/bildungsan gebote/papalapap.
24 Vgl. Verband Bildung und Erziehung. Landesverband NRW und Barth (2005). 


\subsection{Graphem-Phonem-Korrespondenz und lokale Kohärenzbildung}

Bereits im ersten Schuljahr, bei manchen Kindern schon früher, wird ein Verständnis für die Graphem-PhonemKorrespondenz entwickelt, die dann (verbunden mit dem phonologischen Rekodieren) zur Wort- und Satzidentifikation führt (s. Abb. 4). Bis zum dritten Schuljahr werden hier die entscheidenden Fortschritte erzielt. Kinder lernen, (auch ihnen unbekannte) Worte immer rascher zu identifizieren und erbringen damit eine anspruchsvolle Dekodierleistung. Damit einhergehend erweitert sich kontinuierlich der sog. Sichtwortschatz, bis die Worterkennung nahezu automatisch erfolgt (etwa nach dem 2. Schuljahr) und die Wörter nicht mehr mühsam Wort für Wort entziffert werden müssen. Die Kinder erschließen sich zunehmend kürzere, dann längere Sätze. Die Ausbildung dieser Teilkompetenz ist entscheidend, um überhaupt zum flüssigen Lesen zu gelangen. Auf dieser Ebene verarbeitet das Kind den Text noch auf einer ganz oberflächlichen und punktuellen Ebene. Kennzeichnend dafür ist, dass Kinder, die auf diesem Niveau „lesen“ können, es immer noch lieben, vorgelesen zu bekommen, weil ihr Lesevermögen mit ihren Ansprüchen an das eigene Leseerleben nicht mithalten kann.

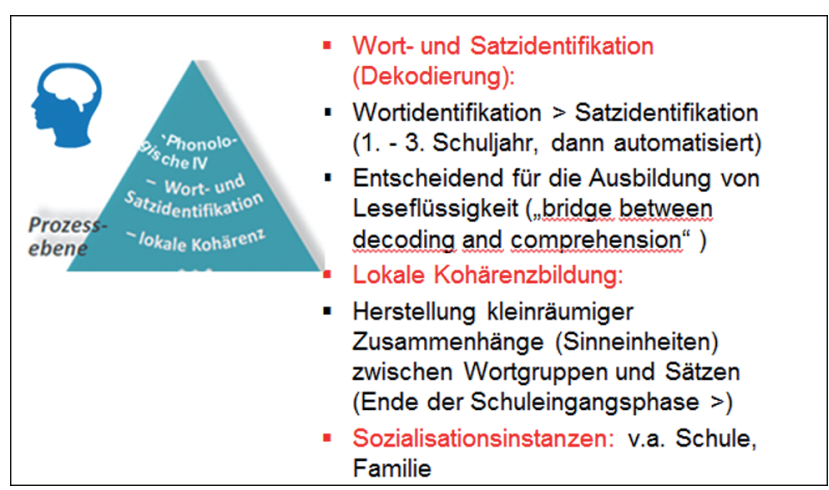

Abb. 4: Leseflüssigkeit und lokale Kohärenzbildung sind erste Schritte zum Textverstehen. Quelle: H.M. Vorlesungsskript

Einhergehend mit dem Flüssiglesen muss eine weitere Leistung erbracht werden. Um einen Text zu verstehen, reicht es i.d.R. nicht aus, nur auf der Ebene von Einzelwörtern oder Wortgruppen zu bleiben. Texte, zumal etwas anspruchsvollere, verlangen, dass Zusammenhänge zwischen Sätzen und Wortgruppen erkannt werden - man spricht von den sog. semantischen Relationen und semantischen Sinneinheiten. Wenn diese semantischen Relationen erkannt werden, können Kinder „lokale Kohärenz“ herstellen, also den Sinn von Texten kleinräumig erfassen und sog. Leerstellen (wenn bspw. eine zusammengehörige Information auf mehrere Sätze verteilt ist), auch unter Rückgriff auf ihr eigenes Weltwissen, füllen und den Text damit verstehen. Auch die Fähigkeit zur lokalen Kohärenzbildung wird durch Lautleseverfahren gefördert.

\subsection{Förderung von Leseflüssigkeit}

Da wir uns beim Flüssiglesen noch auf der Ebene von Messbarem befinden, kann ebenfalls auf eine gesicherte Diagnostik zurückgegriffen werden. Beispielsweise wird das schnelle Identifizieren von Worten mit der Würzburger Leise Leseprobe (1.-4. Schuljahr) oder dem Salzburger Lese-Screening getestet, hier kommt es entscheidend darauf an, dass eine gewisse Geschwindigkeit - Flüssigkeit - erreicht wird. Dies sind immer noch basale Lesefertigkeiten, die aber nun hinführen zu Verarbeitungsprozessen, die hierarchiehöher sind.

Für die Förderung der Leseflüssigkeit werden in erster Linie Lautleseverfahren in den unterschiedlichsten Varianten eingesetzt, weil so die Lesegenauigkeit, der Automatisierungsgrad der Dekodierfähigkeit und die Lesegeschwindigkeit entscheidend verbessert werden können. Lautleseverfahren können einzeln, parallel im Tandem, mit einem versetzt lesenden und kontrollierenden Partnerkind etc. kreiert werden. Sie führen auf einer etwas anspruchsvolleren Ebene hin zu „Vorlesesituationen“, auf die sich Kinder mit wiederholtem Lautlesen vorbereiten (müssen): Formate, mit denen Bibliotheken gängig arbeiten. Hier bleibt die Herausforderung, auch die Kinder zu erreichen, die sich gerade davor scheuen, laut zu lesen ... Das gelingt am besten, wenn Lautlesesituationen geschaffen werden, die eher unkonventionell sind und im Team gemeistert werden (Podcast, Theaterszene mit verteilten Rollen, Radiosendung etc.). ${ }^{25}$ Auch für die Förderung des Flüssiglesens ist Material vorhanden, das kreativ abgewandelt werden kann, insbesondere aus den USA (Abb. 5). In Deutschland gibt es reichlich Material auf den Bildungsservern der Länder (sehr instruktiv bspw. die Materialien auf dem Bildungsserver Berlin-Brandenburg).

25 Das bei Bibliotheken beliebte „Viellesen“ scheint übrigens die Leseflüssigkeit nicht zu fördern. Formate wie der „Sommerleseclub“, die „Antolin-Bücher“, eine Leseolympiade etc. sind dafür weniger geeignet. Vgl. Rosebrock und Nix (2014). 


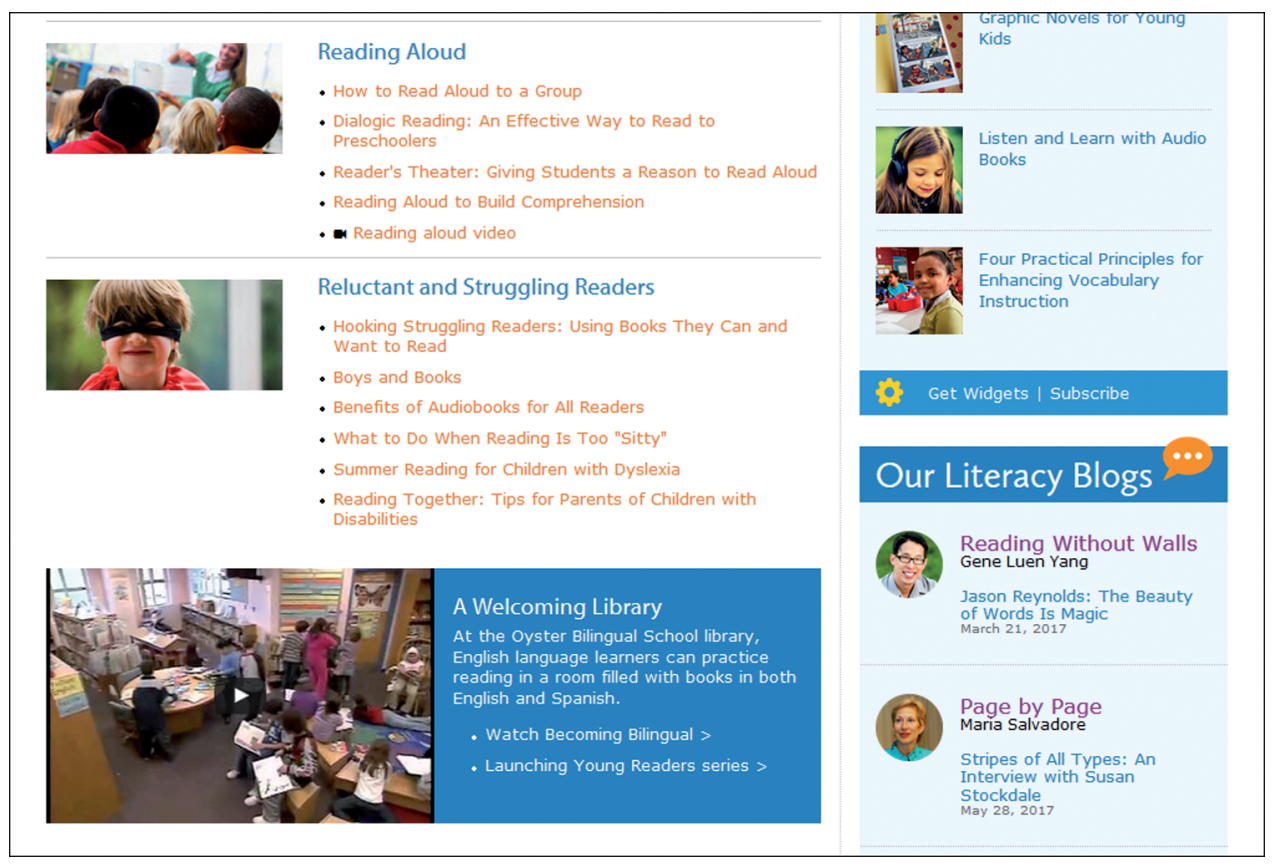

Abb. 5: Die Förderung von Leseflüssigkeit: eine US-amerikanische Initiative (mit vielen Materialien), die von der Library of Congress ausgezeichnet wurde. Diese Seite wendet sich gezielt an Bibliothekare. ${ }^{26}$

\subsection{Herstellen von Globaler Kohärenz}

Eine Vorstellung vom Text als Ganzem zu entwickeln, tatsächlich $\mathrm{zu}$ verstehen, wovon ein auch umfangreicherer Text handelt, ist die nächste Stufe, die nun nicht mehr zu den hierarchieniedrigen Prozessen gehört (s. Abb. 6). Hier muss der Leser eine umfassende Konstruktionsleistung erbringen und Einzelinformationen in einen Zusammenhang bringen, Schlussfolgerungen ziehen usw., folglich globale Kohärenz herstellen. Bedeutsam wird, was er jetzt an eigenem Vor- und Weltwissen, auch eigener Leseerfahrung und damit Lektüreerwartung (bezogen auf den konkreten Text vor sich) aktivieren kann. Guten Lesern gelingt es, diese globale Kohärenz unproblematisch zu bilden, auch, weil sie sich bereits vor (Cover, Inhaltsverzeichnis ...) und bei der Lektüre (Kapitel, Textmerkmale etc.) lesend orientieren und so eine mentale Vorstellung vom vorliegenden Text erhalten und dessen (grobe) Strukturen erfassen. Wenn darüber hinaus poetische Merkmale von Texten oder gar Darstellungsstrategien wahrgenommen und reflektiert werden, sind wir auf der Ebene des literarischen Lesens, das eine Domäne des Literaturunterrichts ist und nicht weiter betrachtet werden soll.

26 http://www.readingrockets.org/audience/professionals/librar ians.

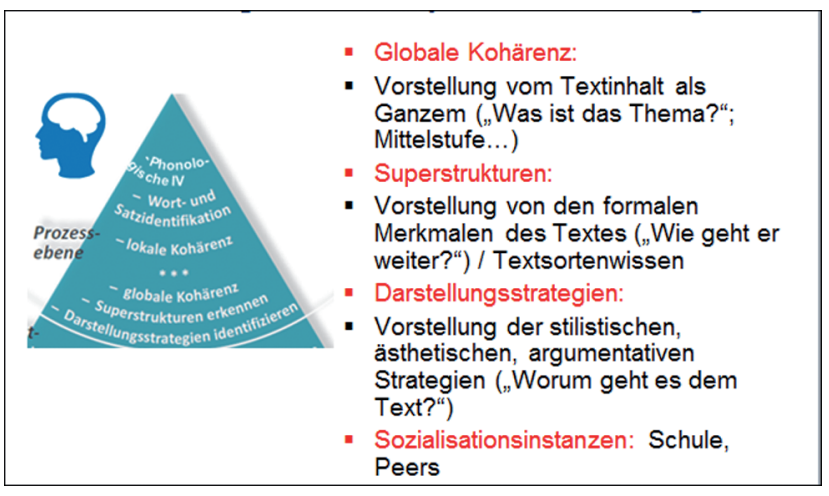

Abb. 6: Ohne globale Kohärenz ist Textverstehen nicht möglich. Quelle: H.M. Vorlesungsskript

Die globale Kohärenz führt erst wirklich zum verstehenden Lesen, insbesondere auch von Sachtexten, die häufig diskontinuierlich, verdichtet und teils formalisiert sind und damit erhöhte Anforderungen an die Verstehensleistung stellen; ungeübte Leser erreichen sie ohne Unterstützung nicht.

Globale Kohärenz kann gefördert werden, indem den Lesenden mentale Werkzeuge an die Hand gegeben werden, mit denen sie sich den Text nähern und ihn erschließen können, die sog. Lesestrategien. Sie zielen darauf ab, das zu herbeizuführen, was bei kundigen Lesern automatisch abläuft: das Aktivieren von Vorwissen (Erwartungen an den Text formulieren), das Wahrnehmen von textlichen Merkmalen (Aufbau, Gliederung), die Identifikation von Schlüs- 
selwörtern, das selbstständige Strukturieren von Textanteilen (Textteile markieren) und vieles mehr. Das kann auch partnerschaftlich oder in Gruppen geübt werden.

Lesestrategien werden zunehmend auch in Schulfächern eingesetzt, bei denen die Ausbildung von Lesekompetenz bislang nicht verortet war. Auch dafür sind in den letzten Jahren zahlreiche Unterstützungsmaterialien erstellt worden. Sehr brauchbar sind bspw. der „Lesepilot“ und der „Lesenavigator“, eine Art Handlungsleitfaden für die Erschließung von Texten für jüngere und ältere Kinder, daneben sind zahlreiche Publikationen erschienen (s. Abb. 7 und 8).

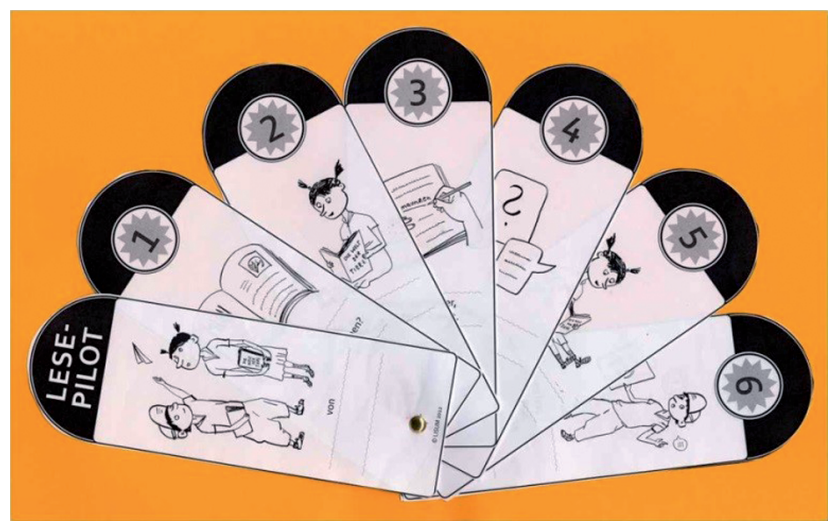

Abb. 7: Der „Lesepilot“ führt jüngere Schüler schrittweise lesestrategisch durch den Text. Quelle: Bildungsserver BerlinBrandenburg ${ }^{27}$

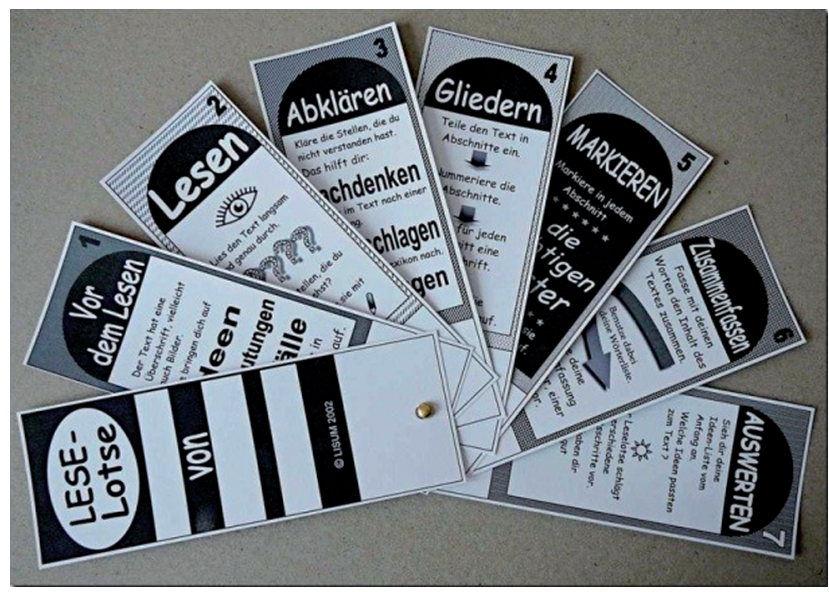

Abb. 8: Der „Leselotse“ unterstützt Schüler beim Erwerb von Lesestrategien $^{28}$

27 http://bildungsserver.berlin-brandenburg.de/themen/sprachbil dung/lesecurriculum/lesen-im-unterricht/lesen-im-deutschunterrich t/lesestrategien/lesepilot/.

28 http://bildungsserver.berlin-brandenburg.de/themen/sprachbil dung/lesecurriculum/lesen-im-unterricht/lesen-im-deutschunterrich $\mathrm{t} /$ lesestrategien/leselotse/.
Nun ist das Lesen gerade nicht zu reduzieren auf die kognitiven Verarbeitungsprozesse. Die beiden unteren Kreissegmente verdeutlichen, dass es immer das Subjekt ist - mit seinem Vorwissen, mit seiner inneren Beteiligung und Motivation - das lesend aktiv ist, das Lesen genießt oder als mühevolle Schwerstarbeit empfindet, das sich in fremde Welten gerne entführen lässt, Vorstellungsvermögen und Empathie lesend entwickeln kann oder entmutigt vor einem Buch kapituliert - das letztlich ein Selbstkonzept als Leser oder eben als Nichtleser ausbildet (s. Abb. 9). Und Bibliothekare wissen, wie wichtig der gesamte soziale Kontext ist, wie unabdingbar positive Leseerfahrungen an soziale Interaktion und Kommunikation, die sog. Anschlusskommunikation, und generell an eine positive soziale Einbettung gebunden sind. Das muss hier nicht herausgestellt werden. Formate, die diese Dimensionen adressieren und fördern, sind vor allem Animationsformate, die zum Lesen verlocken, die Spaß, Genuss und Freude am Lesen wecken sollen - alles Formate, denen Bibliothekare ihre Aufmerksamkeit widmen und die sie erfolgreich realisieren.

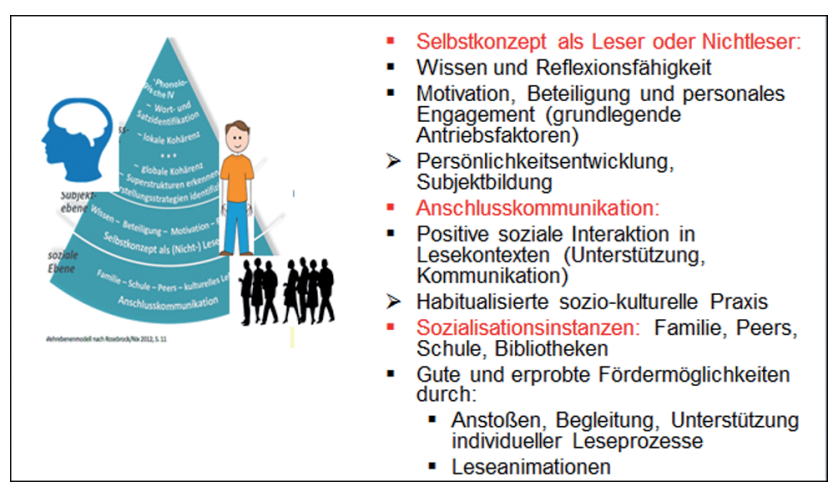

Abb. 9: Die motivationalen und sozialen Dispositionen sind ebenfalls entscheidend für eine erfolgreiche „Lesekarriere“. Quelle: H.M. Vorlesungsskript

Die ketzerische Frage lautet: Reicht das denn nicht aus? Sollen sich Bibliotheken jetzt auch noch mit Leseflüssigkeit, mit Lesestrategien und phonologischen Teilkomponenten beschäftigen? Und verlieren sie damit nicht vielleicht ein Alleinstellungsmerkmal, die motivationale Stärke?

Mit der frühkindlichen Sprach- und Leseförderung sind Bibliotheken ja bereits einen ersten Schritt in genau die Richtung gegangen (wenn auch nicht unbedingt systematisch, s.o.). In der Regel aber erreichen sie mit ihren Aktivitäten bislang die Kinder, die schon gerne, und das heißt auch meistens gut, lesen. Sie erreichen genau diejenigen nicht, deren Probleme auf der Ebene der basalen Lesefertigkeiten beginnen und die sich dann fortsetzen, 


\begin{tabular}{|c|c|c|c|c|}
\hline Stufe & Niveau & Primarbereich & $\begin{array}{l}\text { Sekundarstufel } \\
\text { Mittlerer Schulabschluss }\end{array}$ & \begin{tabular}{|l|} 
Prozessebene \\
(Rosebrock, Nix 2008)
\end{tabular} \\
\hline $\mathbf{I}$ & $\begin{array}{l}\text { Unterhalb } \\
\text { Mindest- } \\
\text { standard }\end{array}$ & $\begin{array}{l}\text { explizit angege- } \\
\text { bene Einzel- } \\
\text { informationen } \\
\text { identifzieren }\end{array}$ & $\begin{array}{l}\text { oberflächliches Verstehen } \\
\text { einfacher Texte, z. B. } \\
\text { auffallige Einzelinformationen } \\
\text { im Text lokalisieren }\end{array}$ & Wort- und Satzidentifikation \\
\hline II & $\begin{array}{l}\text { Mindest- } \\
\text { standard }\end{array}$ & $\begin{array}{l}\text { benachbarte } \\
\text { Informationen } \\
\text { miteinander } \\
\text { verknüplen }\end{array}$ & $\begin{array}{l}\text { Herstellen einfacher } \\
\text { Verknüpfungen, z. B. } \\
\text { - weniger auffallige Einzelinfor- } \\
\text { mationen im Text lokalisieren, } \\
\text { - benachbarte Informationen } \\
\text { und auch Informationen, die } \\
\text { ūber mehrere Abschnitte ver- } \\
\text { teilt sind, miteinander } \\
\text { verknüplen }\end{array}$ & lokale Kohärenz \\
\hline III & $\begin{array}{l}\text { Regelstan } \\
\text { dard }\end{array}$ & \begin{tabular}{|l|} 
"verstreute" \\
Informationen mit- \\
einander verknüp- \\
fen und den Text \\
ansatzweise als \\
ganzen erfassen
\end{tabular} & $\begin{array}{l}\text { Integration von Textelementen } \\
\text { und Schlussfolgerungen aus } \\
\text { komplexeren Texten }\end{array}$ & globale Kohärenz \\
\hline IV & $\begin{array}{l}\text { Regelstan } \\
\text { dard plus }\end{array}$ & \begin{tabular}{|l|} 
für die \\
Herstellung von \\
Kohärenz auf der \\
Ebene des Textes \\
wesentliche \\
Aspekte erfassen
\end{tabular} & $\begin{array}{l}\text { detailliertes Verständnis } \\
\text { komplexer Texte, } \\
\text { z. B. Kombinationen von } \\
\text { Grafiken und kontinuierlichen } \\
\text { Texten }\end{array}$ & Superstrukturen erkennen \\
\hline $\mathbf{v}$ & $\begin{array}{l}\text { Optimal- } \\
\text { bzw. } \\
\text { Maximal- } \\
\text { standard }\end{array}$ & $\begin{array}{l}\text { auf zentrale } \\
\text { Aspekte des } \\
\text { Textes bezogene } \\
\text { Aussagen } \\
\text { selbstständig } \\
\text { begründen }\end{array}$ & $\begin{array}{l}\text { flexible Nutzung unvertrauter } \\
\text { komplexer Texte, z. B.: } \\
\text { Interpretationshypothesen } \\
\text { plausibel beurteilen } \\
\text { Zentrale Thesen aus argumen- } \\
\text { tativen Texten identifzieren }\end{array}$ & $\begin{array}{l}\text { Darstellungsstrategien } \\
\text { identifizieren }\end{array}$ \\
\hline
\end{tabular}

Abb. 10: Die Handreichung für die Entwicklung der Lesekompetenz für die Grundschulen der 1. bis 10. Klasse führt dezidiert die zu erzielenden Teilkompetenzen als zu erreichende Standards auf. ${ }^{29}$

wenn es um das Textverstehen geht. Die deswegen nie ein positives Selbstkonzept als Leser herauszubilden eine Chance haben und die langfristig damit erheblichen Nachteile in ihrer Bildungs- und Erwerbsbiografie zu erwarten haben. Der Anteil dieser Kinder dürfte in den kommenden Jahren nicht geringer werden, im Gegenteil, sie sollten nicht zurückgelassen werden.

Parallel dazu werden die Anforderungen an Kindergärten und Schulen zunehmen, Lesekompetenz für alle Kinder $\mathrm{zu}$ sichern und die geforderten Standards einzulösen (s. Abb. 10). Das wird diese Bildungseinrichtungen schwer fordern, vielleicht überfordern. Es sei denn, sie haben starke Partner - wie die Bibliotheken - die, fachlich kompetent, kundig in Bezug auf die curricularen Kompetenzziele und bereit sind, Leseförderung breiter als bislang zu fassen und entlang der Bildungskette, der kindlichen Entwicklungsetappen und der Teilkompetenzen des Lesens zielgenaue Angebote zu entwickeln. Bibliotheken als relativ schulfer-

29 Vgl. Land Brandenburg (2012). ne und informal agierende Bildungseinrichtungen kann es vielleicht sogar in besonderem Maße gelingen, eine systematische, stringente und umfassende und dennoch motivational positive Leseförderung ins Werk zu setzen.

\section{Literaturverzeichnis}

Artelt, Cordula et al. (2005): Expertise Förderung von Lesekompetenz. Berlin; Bonn: Bundesministerium für Bildung und Forschung (BMBF). Verfügbar unter https://www.bmbf.de/pub/Bildungsfor schung_Band_17.pdf.

Barth, Karlheinz (2005): Früherkennung und Prävention von Lernstörungen. Verfügbar unter www.vbe-nrw.de/vbe_download/gsta k14barth.pdf.

Barth, Karlheinz (2012): Lernschwächen früh erkennen im Vorschulund Grundschulalter. 6., durchges. Aufl. München: Reinhardt.

Barth, Karlheinz (2016): Die Diagnostischen Einschätzskalen (DES) zur Beurteilung des Entwicklungsstandes und der Schulfähigkeit. 7. durchges. Aufl. München: Reinhardt.

Brandt, Susanne; Keller-Loibl, Kerstin (2015): Leseförderung in Öffentlichen Bibliotheken. Berlin; Boston: De Gruyter.

Costard, Sylvia (Hrsg.) (2011): Störungen der Schriftsprache. Modellgeleitete Diagnostik und Therapie. Stuttgart: Thieme. 
Eickelmann, Birgit; Aufenanger, Stefan; Herzig, Bardo (2014): Medienbildung entlang der Bildungskette. Ein Rahmenkonzept für eine subjektorientierte Förderung von Medienkompetenz im Bildungsverlauf von Kindern und Jugendlichen. Deutsche Telekom Stiftung. Verfügbar unter https://www.telekom-stif tung.de/sites/default/files/files/media/publications/buch_me dienbildung.bildungskette_end.pdf.

Garbe, Christine; Holle, Karl; Jesch, Tatjana (2010): Texte lesen. Textverstehen, Lesedidaktik, Lesesozialisation. 2. Aufl. Stuttgart: UTB.

Hellenschmidt, Anja (2015): Bildungspartner Bibliothek - Innovationsbedarf und Selbstwirksamkeit im Berufsfeld der Bibliothekarlnnen. Akzeptanz und Nachhaltigkeit eines Blended-LearningAngebots zur Förderung der Leseförderkompetenz. Dortmund: TU Dortmund.

Hild, Nathalie (2016): Differenzierte Sprachförderung durch Öffentliche Bibliotheken. Wiesbaden: Dinges \& Frick.

Höft, Rita; Marci-Boehncke, Gudrun (2017): Experten für das Lesen. Leseförderung in Öffentlichen Bibliotheken weiterentwickeln. Ein Blick zurück und nach vorn. In: BuB, (1) 51-55.

Krauß-Leichert, Ute; Paul, Jana (2014): Leseförderung - wirkungsvoll oder wirkungslos? Ergebnisse der Studie „Leseförderung und Wirkungsforschung (LeWi)“ der HAW Hamburg und der Stadtbibliothek Bielefeld. Vortrag auf dem 103. Deutschen Bibliothekartag in Bremen, 05.06.2014.

Krautz, Jochen (2016): Kompetenzen machen unmündig. Berlin: GEW. (Streitschriften zur Bildung, 1).

Land Brandenburg (2012): Lesen ist der Schlüssel. Berlin: Land Brandenburg. Ministerium für Bildung, Jugend und Sport. Verfügbar unter https://mbjs.brandenburg.de/media_fast/6288/bro schuere_lesen_ist_der_schluessel.pdf.

Marci-Boehncke, Gudrun; Trapp, Ricarda (2016): Entlang der Bildungskette fördern: Schulische Bibliotheken/Medienzentren zwischen ÖB, WB und dem Habitus eines geübten Lesenden.
Bedarfe - Möglichkeiten - Strategien. 6. Bibliothekskongress Leipzig 14.-17. März 2016.

ProLesen (2011): ProLesen. Auf dem Weg zur Leseschule. Konzepte und Materialien zur Leseförderung als Aufgabe aller Fächer. Schlussbericht der Projektleitung. München. Verfügbar unter http://www.leseforum.bayern.de/download.asp?DownloadFilel $D=09$ bad6 f.6404467f.4bdff102b3b39bd.

Raumel, Frank (2016): Bildungsplan 2016 - Anknüpfungspunkte für geänderte/neue Angebote der Bibliotheken. Vorgehen und erste Beispiele aus dem Medien- und Informationszentrum Stadtbücherei Biberach. Vortrag in Stadtbibliothek Radolfzell: 30.05.2016.

Rose, Stefanie (2013): Bibliothek-Medien-Lesen. Von der Buchausleihe zur Leseförderung: Lesedidaktische Kompetenzen von Bibliotheken im Selbst- und Fremdbild. Eine empirische Studie zu Angebot und Nachfrage außerschulischer Leseförderung in Öffentlichen Bibliotheken unter lesedidaktischer Perspektive. Dortmund: TU Dortmund.

Rosebrock, Cornelia; Nix, Daniel (2014): Grundlagen der Lesedidaktik und der systematischen schulischen Leseförderung. 7. Aufl. Baltmannsweiler: Schneider Verlag Hohengehren.

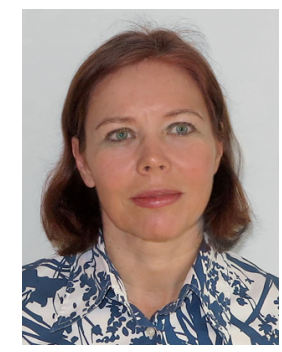

Prof. Dr. Haike Meinhardt

Technische Hochschule Köln Fakultät für Informations- und Kommunikationswissenschaften / Institut für Informationswissenschaft Gustav-Heinemann-Ufer 54 D-50968 Köln haike.meinhardt@th-koeln.de 